\title{
A DESCRIPTIVE STUDY OF INGUINAL HERNIOPLASTY IN A TERTIARY CARE CENTRE
}

\author{
P. Naresh Kumar ${ }^{1}$, Kasinath Panda ${ }^{2}$
}

1Professor, Department of General Surgery, Sri Venkateshwaraa Medical College Hospital and Research Centre, Pondicherry. ${ }_{2}^{2}$ Assistant Professor, Department of General Surgery, Sri Venkateshwaraa Medical College Hospital and Research Centre, Pondicherry.

ABSTRACT

\section{BACKGROUND}

Hernia may be defined as a protrusion of abdominal viscera outside the abdominal cavity through a natural or acquired defect. Inguinal hernias present with a lump in the groin that goes away with minimal pressure or when the patient is lying down. Most cause mild-to-moderate discomfort that increases with activity.

A clinical study on inguinal hernia is undertaken to assess the incidence of inguinal hernia in relation to age, gender and occupation, and to find the complications of inguinal hernioplasty in repairing uncomplicated inguinal hernia by measuring postoperative pain and post-operative complication. Recurrences have been a significant problem following hernia repair. Prosthetic materials have been increasingly used in hernia repair to prevent recurrence.

\section{MATERIALS AND METHODS}

In this descriptive study, 100 tension-free inguinal hernia repairs were performed between June 2015 and April 2017 in 100 patients using a polypropylene mesh (Lichtenstein technique). The main outcome measure was early and late morbidity and especially recurrence.

\section{RESULTS}

Inguinal hernia was indirect in 55\% of cases (55 patients), direct in 30\% (30 patients) and of the pantaloons (mixed) type in $15 \%$ (15 patients). Followup was completed in 80 patients $(80 \%)$ by clinical examination. Seroma and haematoma formation was observed in 6 patients. No acute infection or abscess formation was identified. There was no recurrence of the hernia. Postoperative neuralgia was observed in 5 patients (5\%).

\section{CONCLUSION}

Lichtenstein tension-free mesh inguinal hernia repair is a simple, safe and effective method with low early and late morbidity and low recurrence rate and it is our preferred method for hernia repair.

\section{KEYWORDS}

Lichtenstein's Hernioplasty, Inguinal Hernias, Seroma, Haematoma.

HOW TO CITE THIS ARTICLE: Kumar PN, Panda K. A descriptive study of inguinal hernioplasty in a tertiary care centre. J. Evolution Med. Dent. Sci. 2017;6(73):5242-5247, DOI: 10.14260/Jemds/2017/1138

\section{BACKGROUND \\ Hernias are amongst the oldest known ailments of mankind with the earliest references dating back to 1500 BC. Surgery is the definitive treatment for hernia and remains one of the most commonly performed operations in general surgery worldwide. Inguinal hernias are 9 times more common in males than in females with an overall lifetime risk of developing groin hernias being approximately $15 \%$ in males and $5 \%$ in females. ${ }^{1}$ The incidence of inguinal hernia increases with age from around 11 per 10,000 persons aged between 16 and 24 to around 200 per 10,000 persons aged 75 years and above. ${ }^{2} \mathrm{~A}$ globally aging population means that the demand for surgical services for hernia is ever increasing.}

Financial or Other, Competing Interest: None.

Submission 28-06-2017, Peer Review 26-08-2017,

Acceptance 02-09-2017, Published 11-09-2017.

Corresponding Author:

Dr. Kasinath Panda,

Assistant Professor,

Department of General Surgery,

Sri Venkateshwaraa Medical College

Hospital and Research Centre,

Ariyur-605102, Pondicherry.

E-mail: kasinathpanda@gmail.com

DOI: $10.14260 /$ jemds $/ 2017 / 1138$
The technical aspect of hernia surgery has evolved over time. Numerous techniques have been described to date for the repair of inguinal hernia- both open and laparoscopic. Open method of inguinal hernia repair remains popular worldwide. It can be performed under all types of anaesthesia- general, spinal, epidural and local. Open repair under local anaesthesia has been reported to be both safe and economic. However, its use is not wide spread despite benefits observed by several centres of excellence worldwide. ${ }^{3}$

Inguinal hernia is one of the biggest challenges in surgical practice because of its frequency and complexity. Inguinal hernia repair is the only cure; spontaneous recovery has never been reported. Improved surgical techniques ${ }^{4}$ and a better understanding of the anatomy and physiology of the inguinal canal have significantly improved the outcomes in many patients.

Recurrence following repair of inguinal hernias is a significant problem for both the surgeon and the patient.

\section{Objectives}

1. To compare the clinical presentations of inguinal hernia in all age groups.

2. To know the age and sex distribution of inguinal hernias.

3. To know the post-operative complications and recurrence rate with the hernioplasty. 


\section{MATERIALS AND METHODS}

In this descriptive study, 100 tension-free inguinal hernia repairs were performed between June 2015 and April 2017 in 100 patients using a polypropylene mesh ${ }^{5}$ (Lichtenstein ${ }^{6}$ technique). The main outcome measure was early and late morbidity and especially recurrence.

To find the statistical significance in categorised data, chisquare test was used. Probability value of 0.05 is considered as significant level.

\section{Operative Technique}

The patient is placed in the supine position. The groin is prepared in the usual fashion. Before the incision, a bolus dose of a second-generation cephalosporin is given intravenously. After incising the skin, subcutaneous tissue and external oblique Aponeurosis (as usual), the spermatic cord is elevated from the posterior wall of the inguinal canal. In indirect hernias, the hernial sac is identified, dissected to the internal ring and opened to allow examination of its contents. The sac is ligated and its distal portion is usually excised. However, in large indirect inguinal hernias, where the sac descents down to the scrotum the distal part of the sac may be left open to prevent the formation of a hydrocele, thus allowing spontaneous obliteration. In direct hernias, we prefer to imbricate its contents with non-absorbable sutures (usually polypropylene 2-0). A polypropylene mesh ${ }^{5}(3 \times 5$ inch) is trimmed to fit the floor of the inguinal canal and its apex is first sutured to the pubic tubercle using a No. 3-0 Prolene suture. The same continuous suture then sutured the lower border of the mesh to the free edge of the inguinal ligament, after an opening is made into its lower edge to accommodate the spermatic cord. The continuous suture extends up just medial to the anterior superior iliac spine. Interrupted Prolene ${ }^{7}$ sutures then sutured the two cited edges of the mesh together around the spermatic cord. The inferior medial corner of the mesh is then attached well overlapping the pubic tubercle. The mesh is then anchored to the conjoined tendon by metal staples ${ }^{8}$ (titanium) or by interrupted sutures (prolene 3-0). After meticulous haemostasis, a closed suction drain is placed beneath the external oblique Aponeurosis, especially in large inguinal hernias where an extensive dissection is performed during the plastic reconstruction. The Aponeurosis of external oblique is then closed using absorbable sutures (Vicryl No. 2). Before the closure of the surgical incision, its edges are infiltrated with a long-acting local anaesthetic.

\section{RESULTS}

Inguinal hernia was indirect in $55 \%$ of cases (55 patients), direct in $30 \%$ (30 patients) and of the pantaloons (mixed) type in $15 \%$ (15 patients). Follow-up was completed in 80 patients $(80 \%)$ by clinical examination. Seroma and haematoma formation was observed in 6 patients. No acute infection or abscess formation was identified. There was no recurrence of the hernia. Postoperative neuralgia was observed in 5 patients (5\%).

\begin{tabular}{|c|c|c|c|c|}
\hline \multirow{2}{*}{$\begin{array}{c}\text { Age } \\
\text { Groups }\end{array}$} & \multicolumn{2}{|c|}{ Sex } & \multirow{2}{*}{ Total } & \multirow{2}{*}{ Percentage } \\
\cline { 2 - 4 } & Male & Female & & \\
\hline $0-10 \mathrm{Y}$ & 8 & 1 & 9 & $9.89 \%$ \\
\hline $10-20 \mathrm{Y}$ & 4 & 0 & 4 & $4.40 \%$ \\
\hline $20-30 \mathrm{Y}$ & 12 & 0 & 12 & $13.19 \%$ \\
\hline
\end{tabular}

\begin{tabular}{|c|c|c|c|c|}
\hline $30-40 \mathrm{Y}$ & 11 & 0 & 11 & $12.09 \%$ \\
\hline $40-50 \mathrm{Y}$ & 12 & 0 & 12 & $13.19 \%$ \\
\hline $50-60 \mathrm{Y}$ & 17 & 0 & 17 & $18.68 \%$ \\
\hline $60-70 \mathrm{Y}$ & 18 & 0 & 18 & $19.78 \%$ \\
\hline$>70 \mathrm{Y}$ & 8 & 0 & 8 & $8.79 \%$ \\
\hline Total & $\mathbf{9 0}$ & $\mathbf{1}$ & $\mathbf{9 1}$ & $\mathbf{1 0 0 \%}$ \\
\hline
\end{tabular}

The age distribution of mainly present in $50-60$ years of age $[18.68 \%]$ and mainly affects the males.

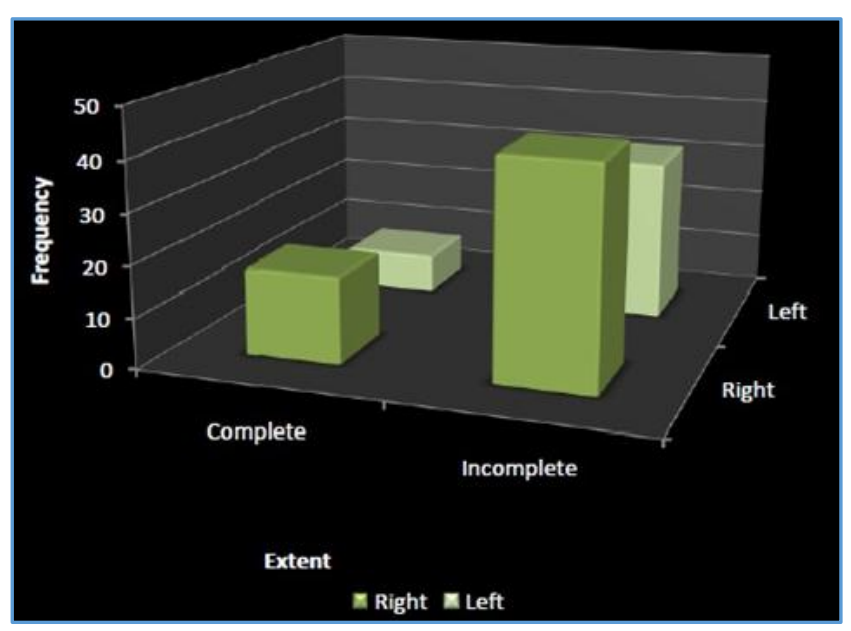

Figure 1. Extent of Hernia

Followup was completed in 80 patients $(80 \%)$ by clinical examination. Seroma and haematoma formation was observed in 6 patients. No acute infection or abscess formation was identified.

There was no recurrence of the hernia. Postoperative neuralgia was observed in 5 patients $(5 \%)$.

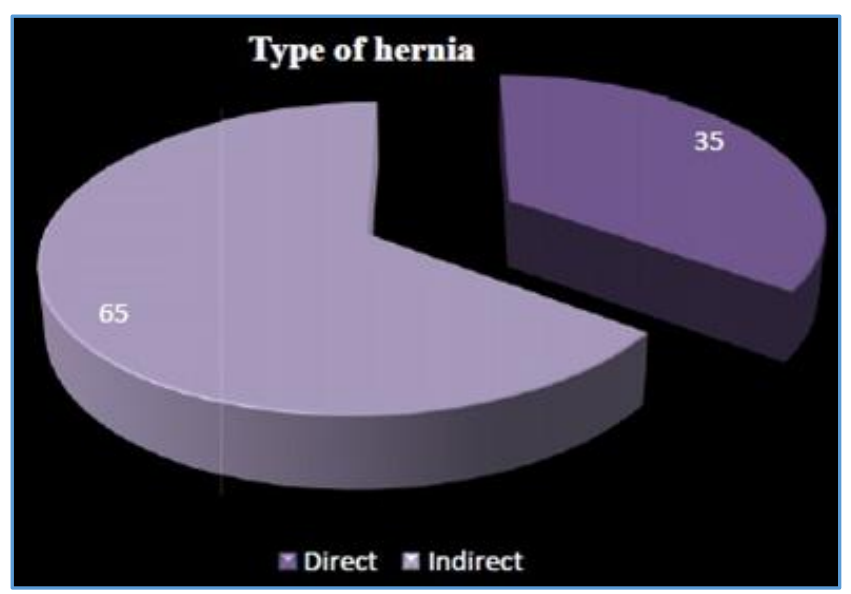

Figure 2. Type of Hernia

\section{DISCUSSION}

\section{The Aetiology of Hernia/ Collagen Theory}

Sir. Arthur Keith stressed the pathological changes in the connective tissues of muscle that leads to its high prevalence in old people. The role of protease-antiprotease imbalance in the pathogenesis of groin hernia has led to a new grasp in the pathology and cause for the surgical failure (recurrence). Adult male inguinal hernias are associated with impaired hydroxylation of prolene, which is aggravated by smoking. These changes cause weakness of fibroconnective tissue of groin and the development of inguinal hernia. Cannon and Read coined this phenomenon as "metastatic emphysema." 


\section{Anatomical or Saccular Theory of Russell}

According to Russell, an inguinal hernia "could not be acquired" but due to the presence of developmental, peritoneal diverticulum which is a necessary antecedent condition in every case of inguinal hernia. This theory could not explain the cause for the absence of hernia in adults with patent processus vaginalis. It is the weakness in the transversalis fascia, which is the prime factor in inguinal and femoral hernia. It is the stretching or distortion of internal ring, which leads to indirect inguinal hernia. Read and his colleagues in 1970 proved the deficiency of collagen content in the recti and transversalis fascia in those with direct hernia.

These observations suggest that the defective synthesis of collagen by fibroblast leads to a quantitative (less than 30\%) and qualitative (defective hydroxylation) deficiency of collagen content in the rectus sheath and transversalis fascia consequently resulting in direct rather than indirect inguinal hernia. Altered collagen metabolism is also seen in Lathyrism, Marfan's syndrome, heavy smoking and Vit. C deficiencies (Brendlen Devlin).

\section{Principal Aetiological Factors \\ Congenital Factors}

1. Sex.

2. Descent of Testicle.

3. Maldevelopment of the abdominal wall.

4. Subtle variants in the attachment and arrangement of abdominal muscle.

\section{Contributing Factors}

1. Ageing.

2. Obesity.

3. Cardiac disease.

4. Pulmonary disease.

5. Prostatism.

6. Constipation.

7. Diverticular disease and colonic carcinoma.

8. Genitourinary tract diseases.

\section{Precipitating or Exciting Causes:}

Sudden intra-abdominal pressure occurs in,

- Coughing.

- Sneezing.

- Straining.

- Heavy weight lifting.

- Crying.

- Trauma.

Injury to ilioinguinal nerve during the surgeries of appendectomy, lumbar sympathectomy, etc.

\section{Sudden Blow Crush Injury}

The conjoint tendon bulges when intra-abdominal pressure rises. Note that damage to the ilioinguinal nerve in the inguinal canal does not paralyse these fibres, since the nerve is purely sensory at this level, having already given off its motor fibres and injury here will only cause some sensory loss over the anterior part of the scrotum (labium majus and adjacent thigh in females).

\section{Congenital Factors}

\section{Descent of Testicle}

Descent of the testis carries with it the tubular projection of peritoneal-processus vaginalis. Patient processus vaginalis along with the enlarged deep inguinal ring cause indirect herniation in infants even with the first cry. The intact internal ring with persistent processus vaginalis does not cause hernia unless it is precipitated by strenuous physical activity causing hernia in adults (Ponka, 1980).

\section{Sex}

Hernia is more common in males than in females. This is probably because of great preponderance of inguinal hernias over all other types.

\section{Maldevelopment of Abdominal Wall and Gastroschisis}

Abnormal development of the abdominal wall results in prompt appearance of hernia; indirect inguinal hernia occurs in subsequent generation also due to genetic determination.

Subtle Variants in the attachment and arrangement of Abdominal Muscles

The female is particularly free from direct inguinal, because the interval between the transversalis arch and inguinal ligament is narrow which protects them from direct herniation (Ponka, 1980). But because of the configuration of the pelvis and the muscular aponeurotic attachment in the women, femoral hernia is more common in them.

\section{Congenital Aetiological Factors in the Development of} Direct Hernia

Congenital disposition of transversalis fascia and Aponeurosis in Hesselbach's triangle causes direct herniation.

\section{Contributing Factors/ Ageing}

Atrophy of tissues leads to muscular weakness of inguinal floor and internal ring leads to inguinal hernia.

\section{Obesity}

Increases intra-abdominal pressure and decreased quality of abdominal musculature by fatty infiltration.

\section{Strenuous Work}

An important contribution factor for direct hernia (Ponka, 1980).

\section{Cardiac Disease}

Leads to congestive cardiac failure with ascites and increased intra-abdominal pressure predisposes to inguinal hernia. Dyspnoea further aggravates the condition.

\section{Pulmonary Disease}

Emphysema, chronic bronchitis, pneumonitis cause chronic coughing which in turn increases intra-abdominal pressure which predisposes to herniation.

\section{Prostatism}

Obstruction to urine flow (secondary to prostatic enlargement) leads to voiding difficulty, which causes increased intra-abdominal pressure. 


\section{Constipation, Diverticular Disease and Colonic} Carcinoma

Results in bowel dysfunction leading to straining of stool repeatedly causing a protrusion to occur.

\section{Genitourinary Disease}

Cystitis, cystocele and urethrocele urethral stricture, phimosis, posterior urethral valve cause difficulty in voiding which leads to increased intra-abdominal pressure.

\section{Anatomy/ Anterior Perspective}

The skin of the lower anterior abdominal wall is innervated by anterior and lateral cutaneous branches of the ventral rami of the lower intercostal nerves and from the ventral rami of the first and second lumbar nerves. The first layers encountered beneath the skin are Camper's and Scarpa's fascia in the subcutaneous tissue. The major blood vessels in this subcutaneous tissue are the superficial epigastric vessels and the superficial circumflex iliac vessels, which are branches of the femoral vessels.

The aponeurosis of the external oblique muscle is the next structure encountered as dissection proceeds through the abdominal wall. The muscle arises from the posterior aspects of the lower eight ribs and interdigitates with both the serratus anterior and the latissimus dorsi at its origin. The posterior portion of the muscle is oriented vertically and inserts on the crest of the ilium.

The anterior portion of the muscle courses inferiorly in an oblique direction toward the midline and pubis.

\section{Inguinal Ligament/ Synonyms}

Crural arch, fallopian arch, femoral arch, arcus inguinalis, Poupart ligament and ligamentum inguinale. The obliquely arranged anterior-inferior fibres of the aponeurosis of the external oblique muscle fold back onto themselves to form the inguinal ligament. It attached laterally to the anterior superior iliac spine. The medial insertion of the inguinal ligament in most individuals is dual. One portion inserts on the pubic tubercle and the pubic bone. The other portion is fan shaped and spans the distance between the inguinal ligament proper and the pectineal line of the pubis.

This fan-shaped portion of the ligament is called the lacunar ligament. (Synonyms: Gimbernat ligament and ligamentum lacunare). It blends laterally with Cooper's (Pectineal) ligament.

The more medial fibres of the aponeurosis of the external or superficial inguinal ring, through which the spermatic cord or round ligament and branches of the ilioinguinal and genitofemoral nerves pass. The internal spermatic vessels (pampiniform venous plexus and the testicular artery) and the genital branch of the genitofemoral nerve, a branch of the lumbar plexus, join the vas deferens at the internal inguinal ring to begin the spermatic cord. The cord structures also include several investing layers. The middle covering layer is called the cremasteric fascia and contains the cremasteric muscle bundles, both of which are derived from the internal abdominal oblique muscle and fascia. The outermost covering of the spermatic cord is the external spermatic fascia, which is continuous with the investing fascia of the external oblique muscle.
The internal oblique muscle lies deep to the external oblique. Its fibres arise from the lateral $2 / 3$ of the grooved upper surface inguinal ligament. Laterally, it is attached to the anterior segment of the iliac crest and posteriorly it is attached to thoracolumbar fascia. The fibres attached to iliac crest diverge and end in the anterior aponeurosis, which gradually broadens from below upwards. The uppermost part of the aponeurosis is attached to the cartilages of 7 th, 8th and 9th ribs.

The important nerves lie in the space between the internal oblique muscle and the external oblique aponeurosis The first lumbar nerve divides into the ilioinguinal and iliohypogastric nerves. These divide between the internal oblique and transversus abdominis muscles. The ilioinguinal nerve may communicate with the iliohypogastric nerve before innervating the internal oblique muscle. The ilioinguinal nerve then passes through the external inguinal ring to run with the spermatic cord, while the iliohypogastric nerve pierces the external oblique to innervate the skin above the pubis. The cremaster muscle fibres, which are derived from the internal oblique muscle are innervated by the genitofemoral nerve (L1, L2).

The transversus abdominis muscle arises from the inguinal ligament, the inner side of the iliac crest, the endoabdominal fascia and the lower six costal cartilages and ribs, where it interdigitates with the lateral diaphragmatic fibres.

The medial aponeurotic fibres of the transversus abdominis contribute to the rectus sheath and insert on the pectin pubis and the crest of the pubis forming the falx inguinalis. These fibres infrequently are joined by a portion of the internal oblique aponeurosis, a true conjoined tendon formed.

The rectus abdominis muscle arises from the fifth to the seventh costal cartilages and inserts on the pubic symphysis and the pubic crest. The lateral edge of the muscle is demarcated by a slight depression in the aponeurotic fibres coursing toward the muscle. This depression is the semilunar line. The arcuate line of Douglas is formed at a variable distance between the umbilicus and the inguinal space. The pyramidalis muscle accompanies the rectus abdominis at its origin in a minority of individuals. It arises from the pubic symphysis and remains within the rectus sheath where it tapers to attach to the linea alba.

In Hesselbach's triangle inguinal ligament forms the base of the triangle, the edge of the rectus abdominis is the medial border and the inferior epigastric vessels are the superolateral border. Hesselbach actually describedCooper's ligament as the base. Deep inguinal ring is situated in the transversalis fascia midway between the anterior superior iliac spine and the symphysis pubis, $1.25 \mathrm{~cm}$ above the inguinal ligament. It is vertically oval in shape. It is related to the arched lower margin of the transversus abdominis and medially to the inferior epigastric vessels.

The inguinal canal is the natural hiatus in the tissues of the anterior abdominal wall. It is obliquely downwards and medially parallel with and little above the inguinal ligament. It extends from deep to superficial inguinal rings. In adults, it is $3-5 \mathrm{~cm}$ in length, bounded anteriorly by the skin, superficial fascia and aponeurosis of external oblique. In its lateral one-third, the anterior wall is reinforced by the muscular fibres of the internal oblique. Posterior to the canal 
lies the reflected inguinal ligament, the conjoint tendon and the transversalis fascia, which separate it from extraperitoneal connective tissue and peritoneum. Superiorly lie the arched fibres of internal oblique and transversus abdominis forming the conjoint tendon. The inferior epigastric vessels are important posterior relations to the medial end of the canal. They lie on the transversalis fascia as they ascend obliquely behind the conjoint tendon into the posterior portion of the rectus sheath.

The endoabdominal fascia is the deep fascia covering the internal surface of the transversus abdominis, the iliacus, the psoas muscles, the obturator internus and portions of the periosteum. It is a continuous sheet that extends throughout the extraperitoneal space and is referred to as the "wallpaper" of the abdominal cavity. The transversalis fascia was first described by Cooper. This is the structure that defines the anterior wall of the preperitoneal space. It is a potential space, relatively avascular and having the appearance of the cotton candy.

The important transversalis fascia analogues are the iliopectineal arch, the iliopubic tract, the crura of the deep inguinal ring and Cooper's (Pectineal) ligament. The superior and inferior crura form a transversalis fascia sling, a "monk's hood" - shaped structure, around the deep inguinal ring. This sling has functional significance, as the crura of the ring are pulled upward and laterally by the contraction of the transversus abdominis, resulting in a valvular action that helps to preclude indirect hernia formation. The iliopubic tract is the thickened band of the transversalis fascia that courses parallel to the more superficially located inguinal ligament. It is attached to the iliac crest laterally and inserts on the pubic tubercle medially. The insertion curves inferolaterally for 1 to $2 \mathrm{~cm}$ along the pectinate line to blend with Cooper's (pectineal) ligament, ending at about the midportion of the superior pubic ramus. Cooper's ligament is actually a condensation of periosteum and is not a true analogue of the transversalis fascia.

Below the iliopubic, the iliopectineal arch separates the vascular compartment that contains the iliopsoas muscle, the femoral nerve and the lateral femoral cutaneous nerve. The vascular compartment is invested by the femoral sheath, which has three subcompartments; the lateral which contains the femoral artery and the femoral branch of the genitofemoral nerve; the middle which contains the femoral vein and the medial the cone-shaped cul-de-sac known as the femoral canal. The femoral canal is normally a 1 to $2 \mathrm{~cm}$ blind pouch beginning at the femoral ring and extending to the level of the fossa ovalis. The femoral ring is bordered by the superior pubic ramus inferiorly and the femoral vein laterally. The iliopubic tract with its curved insertion onto the pubic ramus is the anterior and medial border. The canal normally contains preperitoneal fat, connective tissue and lymph nodes including Cloquet's node at its entrance, the femoral ring. These contents make up the femoral pad, a cushion for the femoral vein allowing expansion as might occur during a Valsalva manoeuvre as well as a plug to prevent abdominal contents from entering the thigh. A femoral hernia exists when the blind end of the femoral canal becomes an opening (the femoral orifice) through which a peritoneal sac can protrude.

The description of the Lichtenstein ${ }^{9}$ tension-free mesh repair, opened a new era in groin hernia repair.
Postoperative pain is minimal as a result of the tension-free technique. The method is very simple, effective, is associated with a very low recurrence rates (ranging from $0 \%$ to $2 \%$ in the literature) and can be performed under local or regional anaesthesia. For these important advantages, it is currently the preferred method for the plastic reconstruction of inguinal hernias for the majority of surgeons around the world.

A variety of prosthetic mesh ${ }^{10}$ is available to the surgeon. The ideal mesh properties are inertness, resistance to infection, molecular permeability, pliability, transparency, mechanical integrity and biocompatibility. Absorbable Mesh does not remain in the wound long enough for adequate collagen to be deposited, while multi-filament mesh can harbour bacteria. Monofilament ${ }^{11}$ mesh is the most popular presently in use with the various types of polypropylene having different characteristic advantages.

Use of porous mesh (Polypropylene) ${ }^{12}$ allows a large surface area for in-growth of connective tissue leading to permanent fixation of the prosthesis within the abdominal wall. Intraparietal placement of the prosthesis allows wellvascularised tissue coverage of all aspects of the prosthesis. Fears of complications related to mesh implantation have proved to be without foundation. The use of vacuum drains is indicated in large inguinal hernias in order to minimise haematoma or seroma formation.

However, duration of antibiotic ${ }^{13}$ use or indication for suction drainage differ among investigators.

To reduce the chance of recurrence, the mesh should extend $2-4 \mathrm{~cm}$ beyond the boundary of Hesselbach's triangle. The position of the mesh beneath the Aponeurosis of the external oblique results in the intra-abdominal pressure working in favour of the repair, since the external oblique Aponeurosis keeps the mesh tightly in place by acting as an external support when intra-abdominal pressure rises. The mesh should be fixated carefully by the use of Prolene ${ }^{14}$ sutures or staples to prevent folding, wrinkling or curling of the mesh around the cord.

The method is simple, can be performed by all the surgeons- even those without special interest in hernia surgery- and is very effective in the prevention of recurrences. Indeed, an extremely low recurrence rate (range, 0\% - 0.7\%) has been reported from many groups of surgeons. The method combines many advantages such as simplicity, effectiveness, safety, comfortable postoperative course with easily controlled pain, rapid return to unrestricted activities, an impressively low recurrence rate and high patient satisfaction. We have been encouraged by the good results of this procedure in a relatively large number of patients.

\section{CONCLUSION}

Lichtenstein's tension-free mesh inguinal hernia repair is a simple, safe and effective method with low early and late morbidity and low recurrence rate and it is our preferred method for hernia repair.

\section{REFERENCES}

[1] Bassini E. Sulla cura radicale dell'ernia injuinale. Arch Soc Ital Chir 1887;4:380-8. 
[2] DeBord JR. The historical development of prosthetics in hernia surgery. Surg Clin North Am 1998;78(6):973-1006.

[3] Usher FC. A new plastic prosthesis for repairing tissue defects of the chest and abdominal wall. Am J Surg 1959;97(5):629-33.

[4] Usher FC, Fries JG, Ochsner JL, et al. Marlex mesh, a new plastic mesh for replacing tissue defects. II. Clinical studies. Arch Surg 1959;78(1):138-45.

[5] Stoppa R, Petit J, Henry X. Unsutured dacron prosthesis in groin hernias. Int Surg 1975;60(8):4112.

[6] Lichtenstein IL, Shulman AG, Amid PK, et al. The tension free hernioplasty. Am J Surg 1989;157(2):188-93.

[7] Popp LW. Endoscopic patch repair of inguinal hernia in a female patient. Surg Endosc 1990;4(1):10-2.

[8] Ramshaw BJ, Tucker JG, Duncan TD. Laparoscopic herniorrhaphy: a review of 900 cases. Surg Endosc 1996;10:255-32.
[9] Kurzer M, Belsham PA, Kark AE. The lichtenstein repair. Surg Clin North Am 1998;78(6):1025-46.

[10] Capozzi JA, Berkenfield JA, Cheaty JK. Repair of inguinal hernia in the adult with prolene mesh. Surg Gynecol Obstet 1988;167(2):124-8.

[11] Amid PK, Shulman AG, Lichtenstein IL. Simultaneous repair of bilateral inguinal hernias under local anesthesia. Ann Surg 1996;223(3):249-52.

[12] Amid PK, Shulman AG, Lichtenstein IL. Open tensionfree repair of inguinal hernias: the lichtenstein technique. Eur J Surg 1996;162(6):447-53.

[13] Goldstein HS. Selecting the right mesh. Hernia 1999;3(1):23-6.

[14] Shulman AG, Amid PK, Lichtenstein IL. A survey of non-expert surgeons using the open tension-free mesh patch repair for primary inguinal hernias. Int Surg 1995;80(1):35-6. 